\title{
THE STUdy ON EleCtRomagnetic SCATTERING CHARACTERISTICS OF JONSWAP SPECTRUM SEA SURFACE
}

\author{
Xiaolin Mi, Xiaobing Wang, Xinyi He and Fei Dai
}

Science and Technology on Electromagnetic Scattering Laboratory, Shanghai, China

\begin{abstract}
The JONSWAP spectrum sea surface is mainly determined by parameters such as the wind speed, the fetch length and the peak enhancement factor. In view of the study of electromagnetic scattering from JONSWAP spectrum sea surface, we need to determine the above parameters. In this paper, we use the double summation model to generate the multi-directional irregular rough JONSWAP sea surface and analyze the distribution concentration parameter and the peak enhancement factor's influence on the rough sea surface model, then using physical optics method to analysis the JONSWAP spectrum sea surface's average backward scattering coefficient change with the different distribution concentration parameters and the peak enhancement factors, the simulation results show that the peak enhancement factor influence on the ocean surface of the average backward scattering coefficient is less than $1 \mathrm{~dB}$, but the distribution concentration parameter influence on the JONSWAP surface of the average backward scattering coefficient is more than $5 \mathrm{~dB}$. Therefore, when we study the electromagnetic scattering of the JONSWAP spectral sea surface, the peak enhancement factor can be taken as the mean value but the distribution concentration parameter have to be determined by the wave growth state.
\end{abstract}

\section{KEYWORDS}

JONSWAP spectrum, multidirectional wave, wave pool, the peak enhancement factor, electromagnetic scattering

\section{INTRODUCTION}

With the in-depth study of sea clutter, the physical quantity of sea spectrum is used to describe the sea surface[1-3]. The sea spectrum is the power density spectrum of the sea surface. It is one of the most basic methods for describing the sea surface. It reflects the statistical distribution of wave energy in the wavelength and propagation direction. It is also the Fourier transform of the sea surface height fluctuation correlation function. The sea-spectrum model can be derived from the equilibrium equation of ocean wave energy, or the autocorrelation function of the sea surface height can be calculated by using fixed-point observation sea surface or laboratory wave-making pool data, and then obtained by Fourier transform. The various forms of sea-spectrum density provided in the existing literature are mostly semi-empirical, semi-theoretical results. Since the 1950s, many oceanographers have done a lot of observations and research on random waves, analyzed various statistical values of ocean waves from the large amount of data obtained, and then selected a function as an approximate expression of the wave spectral density. Common wave spectrums include PM (Pierson-Moscowitz) spectrum, JP (JONSWAP) spectrum, and Wen's spectrum. However, due to the different emphasis of various sea level models, the sea surface generated by different sea level simulations is very different. JONSWAP spectrum is a deep-water wind wave spectrum, which was developed by some institutes of England, Netherland, America and Germany after analyzing and fitting data collected during the "Joint North Sea Wave Observation Project" and is used extensively in the ocean wave research and engineering practice. The JONSWAP spectrum is commonly used for modeling sea surface geometry. 
The JONSWAP spectrum's function is mainly determined by parameters such as wind speed, water depth, and peak enhancement factor. In this paper, we focuses on the effects of different peak enhancement factors and direction concentration parameters on the sea surface electromagnetic scattering. According to the research needs, the multi-directional JONSWAP spectral ocean model is generated by the double stacking method, and the physical optical optics (PO) is used to simulate the sea surface backscattering coefficient under different peak enhancement factors or different direction concentration parameters. Finally, the conclusions of the selection of relevant sea spectrum parameters in the study of JONSWAP sea surface electromagnetic scattering are given.

\section{JONSWAP SPECTRUM}

\subsection{Power Spectrum}

The JONSWAP spectrum function is:

$$
S(\omega)=\frac{\alpha \mathrm{g}^{2}}{\omega^{5}} \exp \left(-\frac{5}{4} \times\left(\frac{\omega_{\mathrm{p}}}{\omega}\right)^{4}\right) \gamma^{\exp \left[-\frac{\left(\omega-\omega_{\mathrm{p}}\right)^{2}}{2 \sigma^{2} \omega_{\mathrm{p}}^{2}}\right]}
$$

Where, $\mathrm{g}$ is acceleration of gravity, $\gamma$ is the peak enhancement factor, which is used to represent wind-wave growth state, its values are in the range of 1.5 6,typical value is $3.3, \omega$ is the wave frequency, $\sigma$ is peak shape parameter , its values are defined by:

$$
\sigma= \begin{cases}0.07, & \omega \leq \omega_{\mathrm{p}} \\ 0.09, & \omega>\omega_{\mathrm{p}}\end{cases}
$$

$\alpha$ is the intensity of the spectrum that relates to the wind speed and fetch length and has the following experience formula:

$$
\alpha=0.076\left(\frac{\mathrm{g} F}{U_{10}^{2}}\right)^{-0.22}
$$

Among them, $\mathrm{F}$ is the wind zone, it is the distance $(\mathrm{km})$ at which the wind blows at a constant speed. $U_{10}$ is the wind speed $(\mathrm{m} / \mathrm{s})$ at $10 \mathrm{~m}$ above sea level. ${ }^{\omega_{\mathrm{p}}}$ is the peak wave-frequency, which is the maximum value appeared in the frequency spectrum. The peak of the JONSWAP spectrum is empirically defined by:

$$
\omega_{\mathrm{p}}=22\left(\frac{U_{10} F}{\mathrm{~g}^{2}}\right)^{-0.33}
$$

From the above analysis, the relationship between the power spectral density and the frequency of the JONSWAP spectrum at a wind speed of $5 \mathrm{~m} / \mathrm{s}$ and a wind region of $40 \mathrm{~km}$ at different peak enhancement factors is shown in Fig. 1. 


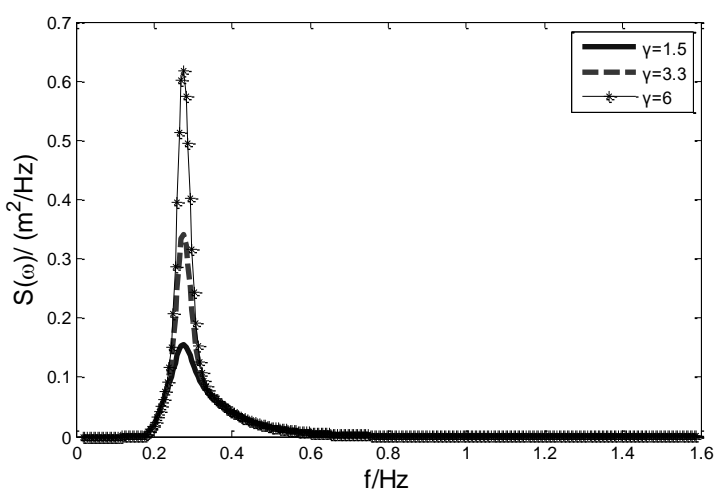

Fig.1 JONSWAP spectrum energy distribution curves with different peak enhancement factors

From Fig. 1 we can see that JONSWAP spectrum is narrow band spectrum, and its energy is mainly focused on some frequency band. The peak enhancement factor in the spectrum is used to correct the shape of the main peak, making the main peak thinner or higher. It can realistically simulate small changes in the wave model.

\subsection{Direction function}

The actual sea surface waves are multi-directional irregular waves, so it is necessary to introduce a direction distribution function [4-9] to describe the energy distribution of the ocean waves with respect to the wind direction. A variety of directional distribution functions have been proposed so far, and the commonly used directional distribution function is an optically easy distribution function.

The directional spectrum is the product of the power spectrum and the direction distribution function, which can be expressed as:

$$
S(f, \theta)=S(f) G(f, \theta)
$$

Where, $S(f)$ is the frequency spectrum, $G(f, \theta)$ is called direction spreading function, the commonly used direction spreading functions are:

$$
G(f, \theta)=G_{0}(s)\left|\cos \frac{\theta-\theta_{0}}{2}\right|^{2 s}
$$

Where $\theta_{0}$ is the main direction of wave propagation, $s$ is the direction distribution concentration parameter, and the coefficient $G_{0}$ is determined by equation (7):

$$
G_{0}(s)=\frac{1}{\pi} 2^{2 s-1} \frac{\Gamma^{2}(s+1)}{\Gamma(2 s+1)}
$$

In the formula, $\Gamma$ is the gamma function. When the direction concentration parameter is independent, we can give out the direction function distribution curves with different direction concentration as in Fig. 2. 


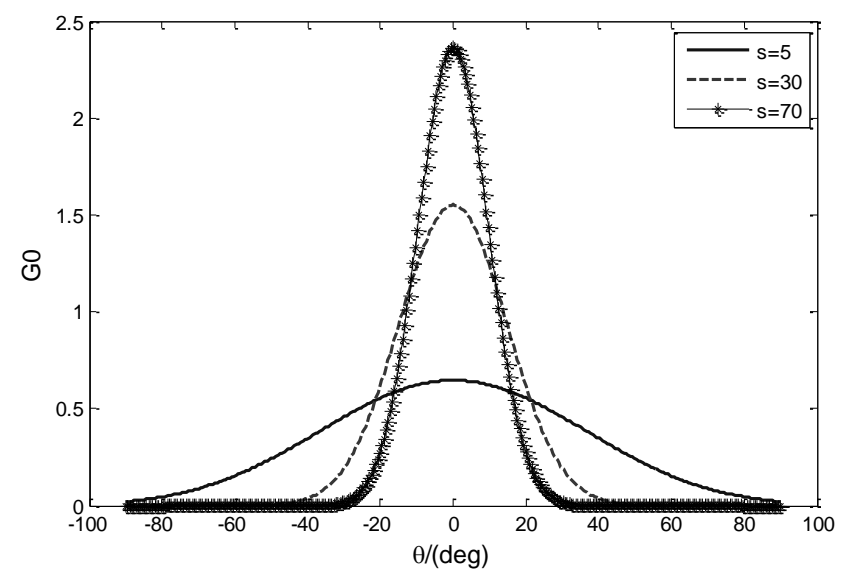

Fig2. The direction function distribution curves with different direction concentration

Figure 2 shows the distribution of the directional function with the direction distribution concentration parameters when the main propagation direction is $0^{\circ}$. It can be seen from the figure that the larger the direction distribution concentration parameter, the more concentrated the wave energy of the multi-directional irregular wave is in the main direction range, that is, the wave energy in the main direction is large, and the wave energy on both sides in the main direction is rapidly reduced. Generally, the wind wave's $s$ is set to about 10 , the swell with a short attenuation distance which $\mathrm{s}$ is set at about 25 , and the swell with a long attenuation distance $s$ is about $75[5]$.

\section{REALIZATION OF Simulating 3D OCEAN WAVES}

Sea waves are of stochastic nature in the stable sea conditions and, mathematically, are represented as Gaussian stationary and ergodic processes. So sea waves can be viewed as wave superposition of infinite simple Cosine waves spreading in the direction of $\theta$ angle relatively with $\mathrm{x}$ axis in $(\mathrm{x}, \mathrm{y})$ plane[9], and those Cosine waves are with different amplitudes, different frequencies and different initial phases. The sea surface elevation $\eta(x, y, t)$ can be represented by the Double Summation Model:

$$
\eta(x, y, t)=\sum_{i=1}^{M} \sum_{j=1}^{N} \zeta_{i j} \cos \left[k_{i}\left(x \cos \theta_{\mathrm{dj}}+y \sin \theta_{\mathrm{dj}}\right)-\omega_{\mathrm{di}} t+\beta_{i j}\right]
$$

Where $k_{i}$ is the wave number $\left(k_{i}=\omega_{\mathrm{di}}^{2} / g\right), \theta_{\mathrm{dj}}$ is spreading directional angle of a single wave $\left({ }^{0<\theta_{\mathrm{dj}} \leq 2 \pi}\right), \omega_{d i}$ is representative frequency in the range of frequency division, $\beta_{\mathrm{ij}}$ is initial phase angle distributed at $\operatorname{random}\left({ }^{0 \leq \theta_{\mathrm{dj}}<2 \pi}\right)$. $\xi_{i j}$ is the wave amplitude of frequency $i$ and directional angle ${ }^{j}$, which can be represented by:

$$
\zeta_{i j}=\sqrt{2 S\left(\omega_{i}, \theta_{j}\right) \mathrm{d} \omega \mathrm{d} \theta}
$$

Equation (8) is the generation principle of multi-directional irregular ocean waves. According to the sea spectrum to be described, the corresponding parameters in the equation are discretized, and the wave height distribution data of the multi-directional irregular sea surface can be obtained through computer simulation. 
According to the above analysis, a multi-directional irregular sea surface of a peak enhancement factor is 3.3 , a wind speed is $5 \mathrm{~m} / \mathrm{s}$ at 10 meters above the sea surface, a direction concentration parameter is 75 and a fetch length is $10 \mathrm{~km}$ is established. The result is shown in Fig. 3.

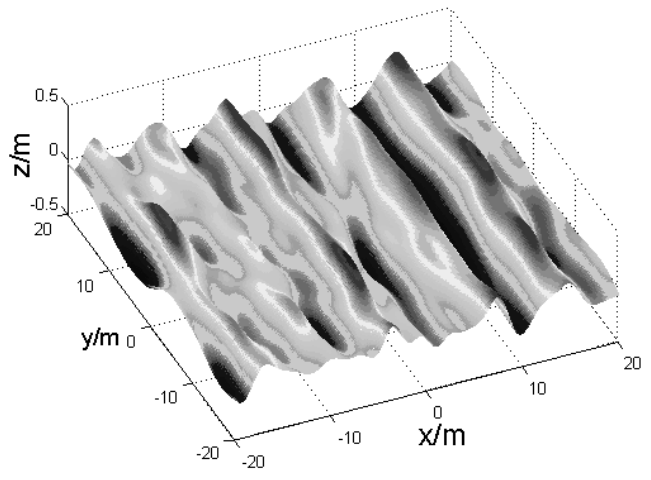

Fig3. The three-dimensional JONSWAP sea surface simulation model

\section{Physical Optics}

The PO method is an approximation method for solving the Helmholtz integral equation. It is widely used in solving electromagnetic scattering problems [10-14]. The basic idea is that when the wavelength of the incident wave and the radius of curvature of the rough surface are satisfied, the rough surface can be regarded as many small triangular facets are spliced together, and the electromagnetic waves are diffracted at the edges and sharp points of the rough surface, and the multiple scattering between the bins can be neglected. The area where the incident wave cannot be directly irradiated is a dark area, and the area where the incident wave can directly illuminate is a bright area, and each bright area scattering field is calculated, and the total scattered field is obtained after superposition. Rough surface element electromagnetic flow is:

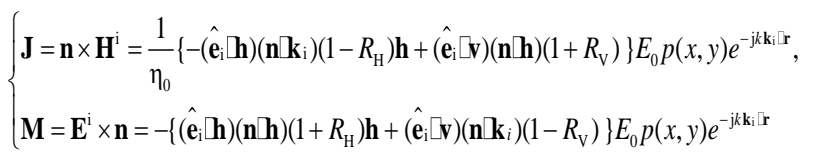

Where, ${ }_{H}, R_{v}$ is the local polarization reflection coefficient, respectively:

$$
\left\{\begin{array}{l}
R_{\mathrm{H}}=\frac{\sin \phi-\sqrt{\varepsilon_{c}-\cos ^{2} \phi}}{\sin \phi+\sqrt{\varepsilon_{c}-\cos ^{2} \phi}} \\
R_{\mathrm{V}}=\frac{\varepsilon_{c} \sin \phi-\sqrt{\varepsilon_{c}-\cos ^{2} \phi}}{\varepsilon_{c} \sin \phi+\sqrt{\varepsilon_{c}-\cos ^{2} \phi}}
\end{array}\right.
$$

Where ${ }^{\phi}$ is the incident angle, which is the complex permittivity, and its calculation formula is :

$$
\varepsilon_{c}=\varepsilon_{r}-\mathrm{j} 60 \lambda \sigma_{e}
$$

Where ${ }^{\varepsilon_{r}}$ is the relative dielectric constant; ${ }^{\sigma_{\mathrm{e}}}$ is the conductivity of the surface material, the unit is Siemens meters ( $\mathrm{S} / \mathrm{m})$.

This gives the scattering field: 


$$
\left\{\begin{array}{l}
\mathbf{E}^{s}(\mathbf{r})=-\mathrm{j} \frac{\omega \mu_{0}}{4 \pi r} e^{-\mathrm{j} k r}\left[\int_{s}\left\{\mathbf{J}\left(\mathbf{r}^{\prime}\right)-\left[\mathbf{J}\left(\mathbf{r}^{\prime}\right) \mathbf{k} \mathbf{k}_{s}\right] \mathbf{k}_{s}+\sqrt{\frac{\varepsilon_{0}}{\mu_{0}}}\left[\mathbf{M}\left(\mathbf{r}^{\prime}\right) \times \mathbf{k}_{s}\right]\right\} e^{\mathrm{j} k r^{i} \mid \mathbf{k}_{s}} d s^{\prime},\right. \\
\mathbf{H}^{s}(\mathbf{r})=-\mathrm{j} \frac{\omega \mu_{0}}{4 \pi r} e^{-\mathrm{j} k r} \succeq \int_{s}\left\{\mathbf{M}\left(\mathbf{r}^{\prime}\right)-\left[\mathbf{M}\left(\mathbf{r}^{\prime}\right) \mathbf{k} \mathbf{k}_{s}\right] \mathbf{k}_{s}+\sqrt{\frac{\varepsilon_{0}}{\mu_{0}}}\left[\mathbf{J}\left(\mathbf{r}^{\prime}\right) \times \mathbf{k}_{s}\right]\right\} e^{\mathrm{j} \mathbf{k}^{i} \mathbf{k} \mathbf{k}_{s}} d s^{\prime}
\end{array}\right.
$$

\section{RESULTS AND COMPARISON}

According to the mathematical model analysis of the first part of JONSWAP spectrum, When the wind speed and wind fetch length are determined, the JONSWAP sea surface is affected by the peak enhancement factor and the direction concentration parameter. The peak enhancement factor mainly represents the power spectrum of the spectral function at the peak frequency, on the geometric model, it shows the sharpness of the waves at the peaks and troughs. The direction concentration parameter mainly represents the concentration of the multi-directional irregular sea surface in the main propagation direction, and the selection of the direction concentration parameter is related to the growth state of the wave. Therefore, in this section, the scattering characteristics of multi-directional irregular ocean waves under different peak enhancement factor and directional concentration parameters are calculated, and their effects on scattering characteristics are analyzed. Thus a method for determining the selection of relevant ocean wave parameters in the simulation or wave-making test of JONSWAP spectrum sea surface when study the electromagnetic scattering is given. During simulation, the dielectric constant of seawater is given according to the double Debye model [14].

\subsection{Influence of peak enhancement factors on electromagnetic scattering characteristics}

When the wind fetch length is $10 \mathrm{~km}$ and the wind speed is $5 \mathrm{~m} / \mathrm{s}$, the JONSWAP spectral dynamic sea surface with peak enhancement factors of 1.5 and 6 is generated respectively. The incident electromagnetic wave frequency is $1 \mathrm{GHz}$, upwind observation, and the sea surface size is $16 \mathrm{~m} * 16 \mathrm{~m}$, and the triangle facet size is smaller than $0.01 \mathrm{~m}$. Under the above conditions, We calculated the backscattering coefficient of the sea surface at the incident angle between $0^{\circ} \sim 70^{\circ}$ by PO. The dynamic sea surface is sampled 40 times at each angle, and the sampling time is $1 \mathrm{~s}$, and the results are the forty average results. When the direction concentration $\mathrm{s}$ is 70 , the curve of the backscattering coefficient under different spectral peak enhancement factors with the incident angle is shown in Fig. 4.

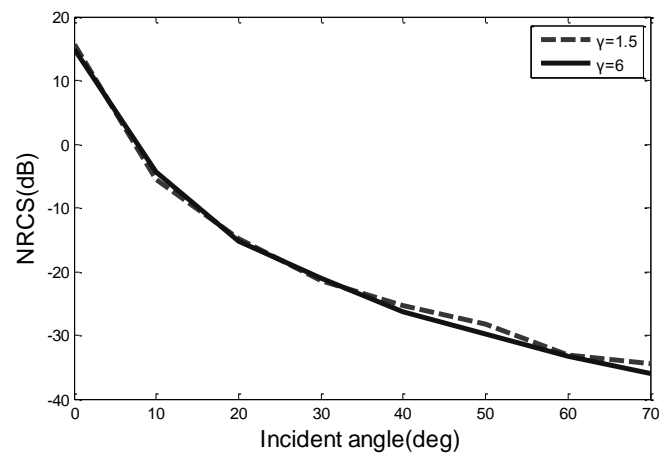

Fig4. The simulation results of normalization radar cross section(NRCS) under different spectral peak enhancement factor 
It can be seen from the figure that the maximum difference of the backscattering coefficient of the multi-directional irregular JONSWAP wave at different incident angles is less than $1 \mathrm{~dB}$ under different spectral peak enhancement factors. Therefore, the peak enhancement factor has little effect on the average scattering coefficient of the ocean wave.

\subsection{Influence of Direction Concentration Parameters on Electromagnetic Scattering Characteristics}

When the peak enhancement factor is the average value of 3.3, the other parameters are the same as those described in Section A. The relationship between the backscattering coefficient and the incident angle under different concentration parameters is simulated as shown in Fig. 5.

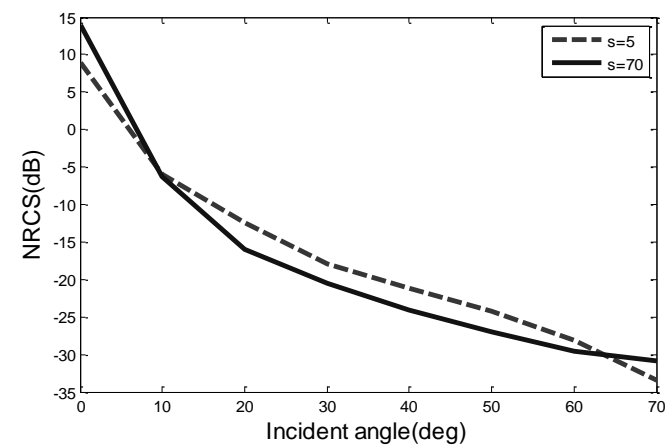

Fig 5. The simulation results of normalization radar cross section(NRCS) under different direction concentration

According to the figure, the maximum difference of the scattering coefficients under different $\mathrm{s}$ parameters exceeds $5 \mathrm{~dB}$. From the geometric model of the sea surface, the larger $\mathrm{s}$ is, the more concentrated the wave is in the main propagation direction. Therefore, the backscattering coefficient of the sea surface is concentrated at the low incident angle to the wind, therefore the result is larger. But as the angle of incidence increases, the back scattering coefficient is smaller than the sea scattering coefficient of the lower s parameter of the coarser distribution.

\section{CONCLuSiON}

In this paper, a Double Summation Model is used to combine the power spectrum and the direction distribution function of JONSWAP spectrum to establish a multi-directional irregular rough sea surface, and the physical optics method is used to simulate the sea surface electromagnetic scattering coefficient. And then give out the conclusions that when the JONSWAP spectrum sea surface is simulated or simulated in the wave pool, the peak enhancement factor parameters have little effect on the electromagnetic scattering characteristics, which can be taken as the average value, but the direction concentration parameter has a great influence on the sea surface electromagnetic scattering, thus its value needs to be determined according to the growth state of the sea surface.

\section{ACKNOWLEDGEMENT}

This work was supported by the National Natural Science Foundation of China (Grant No.61471242). The authors would like to thank the reviewers for their constructive suggestions. 


\section{REFERENCES}

[1] Hasselmann K,Barnett T P, Bouws E,et al. Measurements of wind-wave growth and swell decay during the Joint North Sea Wave Projects (JONSWAP) [J]. Ergnzungsheft zur Deutschen Hydrographischen Zeitschrift Reihe A8(Suppl.),1973,12:95.

[2] Estimation of JONSWAP Spectral Parameters by Using Measured Wave Data[J].China Ocean Engineering,1995(03):275-282.

[3] Annalisa Calini,Constance M. Schober. Characterizing JONSWAP rogue waves and their statistics via inverse spectral data[J]. Wave Motion,2016.

[4] YU Yu-xiu, LIU Shu-xue.Random Wave and Its Applications to Engineering[M],Dalian:Dalian University of Technology Press,2016.

[5] ZHAO Ke, LI Mao-hua, ZHENG JIAN-li, TIAN Guan-nan. 3-D simulation of random ocean wave based on spectrum of ocean wave[J]. Ship Science and Technology,2014,36(02):37-39.

[6] Mitsuyasu $\mathrm{H}$, et al. Observation of the directional wave spectra of ocean waves using a cloverleaf buoy.[J].Physical Oceanography,1975,5:750-760.

[7] Si Liu,Shu-xue Liu,Jin-xuan Li,Zhong-bin Sun. Physical simulation of multidirectional irregular wave groups[J]. China Ocean Engineering,2012,26(3)

[8] Hong Sik Lee,Sung Duk Kim. A three-dimensional numerical modeling of multidirectional random wave diffraction by rectangular submarine pits[J]. KSCE Journal of Civil Engineering,2004,8(4).

[9] MI Xiao-lin, WANG Xiao-bing, HE Xin-yi, XUE Zheng-guo. Simulation and Measurement Technology of 3-D Sea surface in Laboratory Based on Double Summation Model[J].GUIDANCE\&FUZE,2016,37(02):19-23.

[10] WEI Ying-yi, WU Zhen-sen, LU Yue. Electromagnetic scattering simulation of Kelvin wake in rough sea surface[J],CHINESE JOURNAL OF RADIO SCIENCE.,2016,(3): 438-442.

[11] Biglary, H.,Dehmollaian, M.. RCS of a target above a random rough surface with impedance boundaries using GO and PO methods[P]. Antennas and Propagation Society International Symposium (APSURSI), 2012 IEEE,2012.

[12] Joon--Tae Hwang. Radar Cross Section Analysis Using Physical Optics and Its Applications to Marine Targets[A]. Scientific Research Publishing.Proceedings of 2015 Workshop 2[C].Scientific Research Publishing,2015:6.

[13] YANG Peng-ju, WU Rui, ZHAO Ye, REN Xin-cheng. Doppler spectrum of low-flying small target above time-varying sea surface[J]. Journal of Terahertz Science and Electronic Information Technology,2018,16(04):614-618.

[14] MEISSNER T. WENTZ F J. The complex dielectric constant of pure and sea water from microwave satellite observations[J]. IEEE Transactions on Geoscience and Remote Sensing, 2004,42(9): 18361849.

\section{AUTHORS}

Xiaolin Mi (1993-), male, Chinese, engineer, mainly engaged in radar signal processing research.

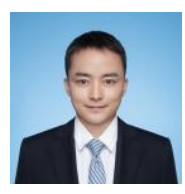

